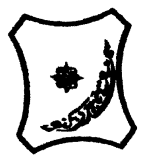

Bayero Journal of Pure and Applied Sciences, 9(2): 40 - 42

Received: July, 2016

Accepted: August, 2016

ISSN $2006-6996$

\title{
PREVALENCE OF Tinea capitis AMONG PRIMARY SCHOOL CHILDREN IN IGALA- MELA/ ODOLU L.G.A, KOGI STATE, NIGERIA
}

\author{
*Yahaya, $\mathbf{O}^{1}$., Yabefa, J.A ${ }^{3}$, Orukotan, A.A. ${ }^{4}$, Jalija, E $^{2}$, Adaja, J.A ${ }^{2}$ and Okpoboh, M.O ${ }^{1}$. \\ ${ }^{1}$ Department of Science Laboratory Technology, Federal Polytechnic, Idah, Kogi State, Nigeria. \\ ${ }^{2}$ Department of Mathematics and Statistics, Federal Polytechnic, Idah, Kogi State, Nigeria. \\ ${ }^{3}$ Department of Science Laboratory Technology, College of Education Technical Brass, Bayelsa State. \\ ${ }^{4}$ Department of Microbiology, Kaduna State University, Kaduna State. \\ *Corresponding author. E-mail: ocholiahmed@yahoo.com
}

\begin{abstract}
A school survey of 1232 primary school children between the ages of 4-12 years was carried out in random/y selected schools in Igala-mela/Odolu L.G.A. of Kogi State for Tinea capitis infection. Cultural and Microscopic examinations of hair and scalp scrapings were carried out. A total of 602 (48.9\%) had the infections, out of which 502 were males and 98 were females (ratio 5:1). Among the isolated dermatophytes, Microsporum canis was most predominant 287(47.7\%), followed by Trichophyton tonsurans 140 (23.3\%),Trichophyton mentagrophytes 112 (18.6\%) while Microsporum audouinit was 63(10.4\%). The high cases of the disease had a strong relationship with low standard of living, poor hygiene, overcrowding and proximity to domestic animals.Personal hygiene, proper sanitation and proper education on handling pet animals are strongly recommended.
\end{abstract}

Key word: Children, Epidemiology,Primary school, Tinea capitis,

\section{INTRODUCTION}

Tinea capitis is a superficial infection of the scalp that occurs mainly in children. The condition is characterized by patches of baldness, erythematous scaling, crusting and black dots produced by breakage of infected hair follicles (Fugueron and Fuller, 1996). The disease is caused by several species of the dermatophytes belonging to the genera Trichophyton, Microsporum and Epidermophyton (Fitzpatrick et al., 1993). The frequent causes of Tinea capitis areT.tonsurans, T.audouinii and M.canis, through humans and animal contacts (Arnold et al., 1990, Hughes et al.,2011).M.ferrugineum and M.gypseum may occationally cause ringworm of the scalp but the endothrix type such as $T$. tonsurans (black dot ring worm) and T.violaceum are most frequent invaders of the scalp and are beginning to replace M.audouinii epidemics of the 50's (Dagnew and Gunther, 1990, Arnold et al., 1990). T.tonsurans regularly affects women adults because of their relationship with children. It has been discovered that $5 \%$ of apparently healthy children are carriers of dermatophytes in their scalp and the main species isolated was T. mentagrophytes (Al-Mosawi et al., 1993; , Binder et al., 2011).

The ectothrix fungi found most frequently on the scalp and the main cause of Kerion is T.verrucosum and T. mentagrophytes. Infection with T.schoenleinii, a relatively common dermatophyte in the middle East, South Africa and Green Land (Yehia, 1980; Kolemen , 1981; Chadegani,1998).Although the initial infection is probably nearly always contracted in childhood, it shows little tendency to clear up spontaneously at puberty and families with several generations affected are often found (Rahim, 1978). T. capitis however, is not a reported diseasebut is a cause for concern because of its contagious nature. T.capitis is considered to be the most frequent cause of dermatophytosis in the republic of Iran and Jordan and the second most frequent cause of dermatophytosis in Mosul (Iraq) after T.corporis (Kolemen,1981; Fathi and Al-Samarai, 2000).

Children are particularly susceptible to T.capitisespecially school children of ages 4 to 14 years because of increased contact than in early childhood. The school surveys are good at measuring the magnitude of the problem. This study is aimed at determining the epidemiological level of the disease among children attending primary schools between ages 4 to 14 and the results will serve as a data base on prevalence of the disease.

MATERIALS AND METHODS

Study Area: Idah is located on the eastern part of the river Niger and bounded on the southern part of the Niger/Benue confluence. It lies on latitude $6^{\circ} 431$ north and longitude 6045 east. The town has two distinct seasons. The rainy season which last from April to September and the dry season which last from October to March. The annual rainfall range, From $140 \mathrm{~cm}$ to $150 \mathrm{~cm}$ a population of 79,815 ( Yahaya et al., 2009).

Sample collection: Specimens were collected randomly from 20 primary schools in Igalamela/Odolu L.G.A. of Kogi State within two academic sessions. Field approach was adopted by visiting school, collecting samples and administering questionnaire forms to children and where respondent could not respond properly, they were followed home for parent'sadvice, and where questions were not understood properly by parents, they were interpreted in their local languages. 
All respondents were either sufferers or hadsuffered the disease earlier.A total of 1232 scrapings were collected and analyzed in Department of Science Laboratory Technology, Federal Polytechnic, Idah. The affected areas were cleansed with $75 \% \mathrm{w} / \mathrm{v}$ ethanol with cotton wool and using sterile blades, scrapings of the scaling, crusting and the dull broken hairs from the margin of the legions were collected in a sterile envelops and carried to the Laboratory with 6 hours of collections for analysis.

Sample analysis: The various specimens were inoculated on sterile culture plates of Sabouraud Dextrose Agar (SDA) that were freshly prepared. The $\mathrm{pH}$ of the culture medium was adjusted to 5.6. and 3 drops of chloramphenicol and $1 \mathrm{ml} / \mathrm{L}$ of Cycloheximide were added to suppress bacteria and fungi other than Tricophytons and Microsporons species. The plates were incubated for 3 weeks and the colonial and morphological appearance of the growth were identified according to the key provided by Collins and Lyne (1979). Test of independence using chi-square analysis and Fishers Exact test at $95 \%$ level of significance was used to test for association of occurrence of the disease with other possible risk factors.

\section{RESULTS}

Out of the 1232 number of samples examined 602 (48.9\%) were positive for the disease, and among these positive cases, Microsporum canis was the prominent species and was found to be $287(47.7 \%)$ followed by Trichophyton tonsurans 140 (23.3\%), Trichophyton mentagrophytes 112 (18.6\%) and Microsporum audouinii 63 (10.4\%) (Table 1).

For the test of independence using the Chi-square contingency Analysis and Fishers Exact Test on the prevalence value,sex had significant influence on the disease incidence, there is higher positive cases in the males than the females $(P<0.001)$. Age of children and the number of children did not show any significant influence on the disease prevalence $(P>0.05)$. The results showed a strong association between the level of education of parents, working class parents and disease occurrence $(P<0.001)$. There is no association between bed sharing and disease incidence $(P>0.05)$, there was significant association between source of water, irregular bathing habit and the disease $(\mathrm{P}<0.001)($ Table 2$)$.

Table 1. isolated species of Tinea capitisand their prevalence rate in the study population.

\begin{tabular}{llc}
\hline Genera & \multicolumn{3}{l}{ No. of cases } \\
\hline Microsporum canis & 287 & $(47.7)$ \\
Microsporum audouinii & 63 & $(10.4)$ \\
Trichophyton tonsurans & 140 & $(23.3)$ \\
Trichophyton Mentagrophyte & 112 & $(18.6)$ \\
\hline
\end{tabular}

Table2. Distribution of the disease and common factors that could contribute to the spread Tinea capitis among the school children.

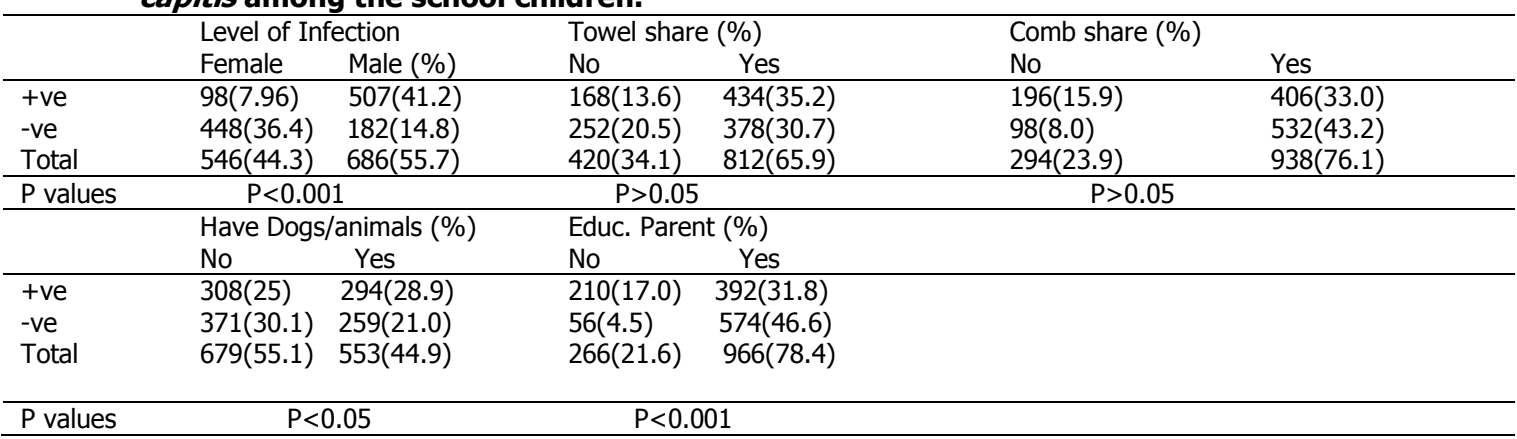

\section{DISCUSSION}

One of the greatest problems hindering the eradication and prevention of Tinea capitis is the presence of healthy, asymptomatic dermatophytes carriers. In this study the prevalence rate is higher (48.9\%)compared to the results (9\%) from 5 primary schools in Bengzazi, a rural Kuwait (Al-Mosawi et al., 1993). Ajao and Akintunde (1985)found a prevalence rate for clinical infections among school children in Ile Ile to be $14.02 \%$ and in urban and rural school in and around Lusaka was $16.8 \%$ (Simpanya,1989), and in Sarajevo it was $52.6 \%$ of the children that were infected (Prophic, 2008).

The relatively high rate of the infection in the community surveyed could be attributed to lack of adequate information on the disease and the possible misconception about the cause, low standard of living, overcrowding and poor hygiene as reason for the apparent increase in the spread of the disease.

In this work, more males were infected (Table1) the reason for this can be attributed to the easy implantation of the spores due of short hairs, frequency of sharing combs, brushes, caps and visits to village barbers whose unhygienic practices may lead to the transmission of infections from persons to persons. Tinea is a prepubertal disease and it was found that higher rate of infection is in children under 11 years of age. In previus reports, Yehia (1980), Raccurt et al., (2009), Jankowska-Konsur et al.,(2009) higherprevalence of Tinea capitis in childrenbetween $8-12$ years is $53.8 \%, 63.2 \%, 70 \%$ were recorded respectively. 
London had a frequency of $71.3 \%$ of scalp infections in male and $28.7 \%$ in females with children in the majority, in Sarajevo the infection is more in male children less than 10years of age (56.5\%) (Prophic, 2008). The deficiency of sebum which acts as a fungistic factor will favor infectionand the presence of dermatophytes on healthy skin may be due to commensalisms. Factors such as high blood sugar level and the presence of fatty acid in skin may determine the presence of these organisms and explain their gradual decline with advancing age (Clayton 1977; Martinez, 1980 ).

Contact with animals is considered a risk factor for Tinea capitis and $11 \%$ of the subjects have dogs and play with other domestic ruminant animals. In other related studies, $82.5 \%$ of positive cases in primary school children have high evidence of contact with cattle, sheep, chickens and stray dogs. Animal ring worm may be acquired both through direct contacts

\section{REFERENCES}

Ajao, A, O. and Akintunde, C. (1985). Studies on the prevalence of Tinea capitis in Ile-Ile, Nigeria. Mycopathologia, 89(1): 43-8.

Al-Mosawi, T., Al-Affas N. H. and Al-Ramahya, A. K. (1993). The incidence of scalp fungal infestation among primary pupils in Basrah city. Journal of community Medicine. 6:31-6.

Arnold, H. L., Odom, R. B. and James, W. D. (1990). Andrew's disease of the skin. $8^{\text {th }}$ edition, Philadelphia, W D Saunders Company. Pp 15-17.

Binder, B., Lackner, H.K., Poessl, B.D., Propst, E., Weger, W., Smolle, J. and GinterHanselmayer, G. (2011). Prevalence of Tinea capitis in Southeastern Austria between 1985 and 2008. Mycosis. 54(3) 243-7

Chadegani, M. (1998). A study of dermatophytosis in Esfahan (Iran), Mycopathologia. 98(2):1014.

Clayton, Y. M. (1977). Epidemiological Aspects of dermatophytes infections: current therapy research, British Journal of Medicine. 22(1):18-22.

Collins, C. H. and Lyne, P. M. (1979). Microbiological Methods. $4^{\text {th }}$ edition, Butler Worth, London. Pp 489-495.

Dagnew, M. B. and Gunther, E. (1990). Epidemiology of communicable skin disease in children of rural area in Northern Ethiopia. Dermatologische Monatsschrift. 176 (4) 21923.

Fathi, H.I. and Al-Samarai, A.G.M. (2000)Prevalence of Tinea capitis among schoolchildren in Iraq. East. Med. Health J. 6 (1):128-137.

Figueron, J.L and Fuller, L.C. (1996). The prevalence of skin disease among school children in rural Ethiopia. Paediatric Dermatology. 13(5): 378-81.

Fitzpatrick, T.B. (1993) Dermatology in general medicine, $4^{\text {th }}$ edition, New York, McGraw Hill.pp288-300.

Hughes, R., Chiaverini, C. and Bahadoran, P. (2011). Corkscrew hair: a new dermoscopic sign for with infected animals. Or indirectly through formites and a significant number of children with Tinea capitis are involved in the rearing of animals (Jones, 1995; Sahgalet al., 1985, Raccur et al.,2009, JankoowskaKonsur et al.,2009).

T. capitis infection is also linked to overcrowding, the results of this work showed that most homes have an average of 4-5 children leading to frequent contacts between family members. Other factors like source of water, bathing habits, types of soap and cream and sharing of towels (general hygiene) had a strong association with the disease incidence in this study.

\section{CONCLUSION}

The magnitude of the disease among the tested age group is high and is becoming a re-emerging disease and efforts should be made towards eliminating the risk factors. Early detection and treatment of infected persons are strongly recommended.

diagnosis of Tinea capitis in black children. Arch. Dermotol. 147(3):355-6.

Jankowska-Konsur, A., Dylag, M. and Szepietowski, J.C. (2009). Tinea capitis in Southwest Poland. Mycosis. 52(2):193-4.

Jones, T.C. (1995). Overview of the use of terbinafine (Lamisil) in children. British journal of Dermatology. 132:683-689.

Kolemen, F. (1981). Dermtophytic flora of Ankara (Turkey). Dermatologia. 162(4):260-4.

Martinez, R. L. (1980). Isolation of dermatophytes from different natural sources. Mycoses. 396:205-10.

Prophic, A. (2008). An epidemiological survey of Tinea capitis in Sarajevo, Bosnia and Herzegovinia over a 10 years period. Mycosis 51 (2):1614.

Raccurt, C.P., Dorsainvil, D., Boncy, J. and Auguste, G. (2009). The emergence of Tinea tonsurans in Port-au-prince, Haiti. Med. Mycol. 47(2):197-200.

Rahim, G. F. (1978). A survey of fungal causing Tinea capitis in Iraq. British Journal of Dermatology. 4:213-8.

Sehgal, V. N., Sexena, A.K. andKumari, S. (1985). Tinea capitis. A clinicoetiologic correction. International Journal of Dermatology.24(2):116-9.

Simpanya, M. F. (1989). A contribution to the study of Tinea capitis in Lusaka, Zambia. East African Medical Journal. 66 (4): 269-75.

Yahaya, O., Miachi, O.E., Umar, I.O and E. Uwaokhonye (2009) Malaria Parasitaemia among Pregnant Women with Multiple Child Birth Attending AnteNatal Clinics in Parts of Idah and Igalamela/Odolu Local Government Areas of Kogi State, Nigeria. International Journal of Medicine and Medical Sciences 1(11) 527- 529.

Yehia, M. M. (1980). Studies on Dermatophytes in Mosul and Vicinity (thesis), University of Mosul, Collage of Medicine. Pp48-106. 